\title{
Impact of World Health Organisation's guidelines for the treatment of pain and the European Medicines Agency's safety recommendations on non-steroidal anti-inflammatory drugs use in analgesics utilization in Lithuania and other European countries. Observational study
}

\author{
Skaiste Kasciuskeviciute, Gintautas Gumbrevicius, Kestutis Petrikonis, Edmundas Kadusevicius
}

\section{Lithuanian University of Health Sciences, Lithuania}

Background and objective: Irrational nonsteroidal antiinflammatory drugs (NSAIDs) use is the main cause of adverse effects associated hospitalisations among all medicines leading to extremely increased costs for health care. Undoubtedly, measures should be taken to prevent it. An important step is pharmacoepidemiological studies which can reveal predominant problems and encourage further decisions. So, our purpose was to study the utilization of nonopioid analgesics (ATC codes N02B; M01A) and to analyse compliance with World Health Organisation's (WHO) guidelines for the treatment of pain and the European Medicines Agency's (EMA) safety recommendations in Lithuania comparing to other European countries.

Materials and methods: The defined daily dose (DDD) methodology was applied to assess analgesics, classified in N02B and M01A, utilization, expressed in DDDs/1000 inhabitants/day.

Results: Over the 11-year period, the utilization of N02B and M01A increased by $22.8 \%$ in Lithuania. Contrary to WHO recommendations on pain management, all Baltic countries were more likely to use NSAIDs than other analgesics and antipyretics: in 2015, NSAIDs were used 6.04; 5.79 and 6.11 times more than those of N02B in Lithuania, Estonia and Latvia, respectively, whereas Scandinavian countries prefered N02B to M01A group: in Denmark utilization of other analgesics and antipyretics was 2.33 times higher than NSAIDs, in Sweden - 1.24 times higher. In Norway, the use of both groups was similar. In Scandinavian countries paracetamol was the analgesic of first choice, whereas in Lithuania it took only the third place. Although EMA has restricted the use of certain NSAIDs - COX-2 inhibitors, nimesulide and diclofenac - their use has consistently increased (15.91; 2.83 and 1.41 times respectively) showing incompliance with international guidelines.

Conclusions: Neither EMA's safety policy nor WHO guidelines for the treatment of pain do not have enough impact on rational NSAIDs use in Lithuania. Prevalence of EMA's restricted NSAIDs use remains high or even increases. Incompliance with international guidelines may result in increased morbidity, mortality and higher costs for health care. Taking into account issues highlighted in this observational study, national recommendations which aim for rationalization of non-opioid analgesics use were developed. 